\title{
Neural-Network-Based Time-Delay Estimation
}

\author{
Samir Shaltaf \\ Department of Electronic Engineering, Princess Sumaya University for Technology, P.O. Box 1438, \\ Al-Jubaiha 11941, Amman, Jordan \\ Email: shaltaf@psut.edu.jo
}

Received 4 May 2003; Revised 13 August 2003; Recommended for Publication by John Sorensen

\begin{abstract}
A novel approach for estimating constant time delay through the use of neural networks (NN) is introduced. A desired reference signal and a delayed, damped, and noisy replica of it are both filtered by a fourth-order digital infinite impulse response (IIR) filter. The filtered signals are normalized with respect to the highest values they achieve and then applied as input for an NN system. The output of the $\mathrm{NN}$ is the estimated time delay. The network is first trained with one thousand training data set in which each data set corresponds to a randomly chosen constant time delay. The estimated time delay obtained by the NN is an accurate estimate of the exact time-delay values. Even in the case of noisy data, the estimation error obtained was a fraction of the sampling time interval. The delay estimates obtained by the NN are comparable to the estimated delay values obtained by the cross-correlation technique. The main advantage of using this technique is that accurate estimation of time delay results from performing one pass of the filtered and normalized data through the NN. This estimation process is fast when compared to the classical techniques utilized for time-delay estimation. Classical techniques rely on generating the computationally demanding cross-correlation function of the two signals. Then a peak detector algorithm is utilized to find the time at which the peak occurs.
\end{abstract}

Keywords and phrases: Neural networks, time-delay estimation.

\section{INTRODUCTION}

Time-delay estimation problem has received considerable attention because of its diverse applications. Some of its applications exist in the areas of radar, sonar, seismology, communication systems, and biomedicine [1].

In active source location applications, a continuous reference signal $s(t)$ is transmitted and a noisy, damped, and delayed replica of it is received. The following model represents the received waveform:

$$
r(t)=\alpha s(t-d)+w(t)
$$

where $r(t)$ is the received signal that consists of the reference signal $s(t)$ after being damped by an unknown attenuation factor $\alpha$, and delayed by an unknown constant value $d$, and distorted by additive white Gaussian noise $w(t)$.

A generalized cross-correlation method has been used for the estimation of fixed time delay in which the delay estimate is obtained by the location of the peak of the crosscorrelation between the two filtered input signals $[2,3]$. Estimation of time delay is considered in $[4,5]$, where the least mean square (LMS) adaptive filter is used to correlate the two input data. The resulting delay estimate is the location at which the filter obtains its peak value. To obtain the noninteger value of the delay, peak estimation that involves inter- polation is used. Etter and Stearns have used gradient search to adapt the delay estimate by minimizing a mean square error, which is a function of the difference between the signal and its delayed version [6]. Also, So et al. minimized a mean square error function of the delay, where the interpolating sinc function was explicitly parameterized in terms of the delay estimate [7]. The average magnitude difference function (AMDF) was also utilized for the determination of the correlation peak [8]. In [8], During has shown that recursive algorithms produce better time-delay estimate than nonrecursive algorithms. Conventional prefiltering of incoming signals used in [2] was replaced by filtering one of the incoming signals using wavelet transform [9]. Chan et al. in [9] has used the conventional peak detection of the crosscorrelation for estimating the delay. Chan et al. reported that their proposed algorithm outperforms the direct crosscorrelation method for constant time-delay estimation. In [10], Wang et al. has developed a neural network (NN) system that solves a set of unconstrained linear equations using $L_{1}$-norm that optimizes the least absolute deviation problem. The time-delay estimation problem is converted to a set of linear algebraic equations through the use of higherorder cumulants. The unknown set of coefficients represent the parameters of a finite impulse response filter. The parameter index at which the highest parameter value occurs represents the estimated time delay. The algorithm proposed by Wang et al. is capable of producing delay estimates; this 
algorithm produces only a multiple integer of sampling interval and does not deal with the case of fractional time delay. Also, the Wang et al. algorithm has utilized the high-order spectra, which requires heavy computational power.

In this paper, direct estimation of constant time delay is accomplished through the use of NN. The NN has proven to be powerful in solving problems that entail classification, approximation of nonlinear relations, interpolation, and system identification. It has found its way into many applications in speech and image processing, nonlinear control, and many other areas.

In this paper, the capability of generalization and interpolation of the NN is employed in the area of time-delay estimation. The NN is trained with many different sets of data that consist of the reference signal and its damped, delayed, and noisy replica. A fourth-order type II Chebyshev bandpass digital filter is used to filter the two signals. The filtered signals are normalized with respect to the highest values they achieve before they are applied to the NN input. The noisefree reference signal is filtered to make it experience the same transient effect and phase shift the filtered delayed noisy signal has experienced.

The rest of this paper is organized as follows. Section 2 presents the details of the proposed technique of using the $\mathrm{NN}$ for the estimation of constant time delay. Section 3 presents the simulation results of the proposed technique. The conclusion is presented in Section 4.

\section{TIME-DELAY ESTIMATION ALGORITHM}

The reference signal $s(t)$ is assumed to be a sinusoidal signal with frequency $\Omega_{o} \mathrm{rad} / \mathrm{s}$, and sampled with a sampling period $T$ seconds. The resulting discrete reference signal is

$$
s(n)=\sin \left(\omega_{o} n\right),
$$

where $\omega_{o}=\Omega_{o} T$ is the frequency of the sampled reference signal. Assuming the received signal in (1) has been filtered by an antialiasing filter and then sampled, then its discrete form is

$$
r(n)=\alpha s(n-D)+w(n),
$$

where $s(n-D)$ is the delayed reference signal, $D$ is an unknown constant delay measured in samples and related to the time delay $d$ by the relation $D=d / T, \alpha$ is an unknown damping factor, and $w(n)$ is zero-mean white Gaussian noise with variance $\sigma_{w}^{2}$. Finite length data windows are obtained from the sampled reference signal and the damped, noisy, and delayed signal with a length of $N$ samples.

Let $\mathbf{s}=[s(0), s(1), \ldots, s(N-1)]$ and $\mathbf{r}=[r(0), r(1)$, $\ldots, r(N-1)]$ be the vector forms of the two data windows representing the reference and the received signals, respectively. Since the received signal is noisy, it is best that it gets filtered in order to obtain better estimate for the unknown time delay.

A fourth-order type II Chebyshev bandpass digital filter was used to filter the two signals. The filter was designed to have a narrow pass bandwidth equal to 0.02 radian with $2 \mathrm{~dB}$ attenuation for the two pass frequencies and a stop band bandwidth of 0.32 radian with a $40 \mathrm{~dB}$ attenuation for the stop frequencies. The center frequency $\omega_{c}$ of the bandpass filter was set equal to the reference signal frequency $\omega_{o}$, and was set to be exactly equal to the geometric mean of the pass and stop frequencies. The resulting bandpass digital filter order that satisfies the above conditions was found to be 4 . The strict narrow bandwidth condition resulted in a bandpass filter that was capable of reducing the input noise power to about $1.6 \%$ of its value at the filter output. This means a noise reduction factor equal to 62.5 , which implies a signalto-noise ratio improvement by $18 \mathrm{~dB}$. This improvement on the signal-to-noise ratio results in improving the accuracy of the time-delay estimates.

Let the filter frequency response be $H(\omega)$ and assume that the filter input is a white Gaussian noise $w(n)$ having zero mean and variance $\sigma_{w}^{2}$. Let the filter output be $y(n)$. The output signal $y(n)$ will then be a zero-mean colored Gaussian noise with variance $\sigma_{y}^{2}$, where

$$
\sigma_{y}^{2}=\frac{1}{2 \pi} \int_{-\pi}^{\pi} \sigma_{w}^{2}|H(\omega)|^{2} d \omega .
$$

The frequency response $H(\omega)$ of the bandpass filter was obtained through the Matlab environment. The white Gaussian noise $w(n)$ was assumed to have a unit variance $\sigma_{w}^{2}=1$. The output signal variance $\sigma_{y}^{2}$ was calculated through the Matlab to have a value of 0.016 .

The bandpass filter is used to filter the received noisy signal and improve the signal-to-noise ratio which is a needed step before applying the signal to the NN. The reference signal $s(n)$ is also filtered by the same filter to make it experience the same effect the received filtered signal has experienced. The two signals are now in perfect match with each other except for the unknown constant time-delay value $D$ and the damping factor $\alpha$. To eliminate the effect of the presence of the damping factor $\alpha$, the two filtered signals are normalized with respect to the highest values they achieve. Normalizing the filtered signals is used to prevent the $\mathrm{NN}$ from being affected by the different amplitude variations of the signals due to the different and unknown damping factors. The signalto-noise ratio which is a very important determining factor of the accuracy of the time-delay estimate is not affected by the normalization step. By applying the filtered and normalized signals to the NN, the NN should then be capable of producing accurate time-delay estimates. If the received noisy signals were used instead, the NN would produce time-delay estimates which are far from being accurate.

Let $h(n)$ be the impulse response of the filter and apply the signals $s(n)$ and $r(n)$ as inputs to the filter; the corresponding outputs are

$$
\begin{array}{ll}
s_{f}(n)=\sum_{k=0}^{n} h(k) s(n-k), & n=0, \ldots, N-1, \\
r_{f}(n)=\sum_{k=0}^{n} h(k) r(n-k), & n=0, \ldots, N-1,
\end{array}
$$


TABLe 1: Parallel input mode (exact delay $d=0.153$ second, damping factor $\alpha=1$ ).

\begin{tabular}{|c|c|c|c|c|c|c|c|}
\hline & \multirow{2}{*}{ Neural Network } & \multicolumn{2}{|c|}{ Noise SD $=0.1$} & \multicolumn{2}{|c|}{ Noise $\mathrm{SD}=0.3$} & \multicolumn{2}{|c|}{ Noise $\mathrm{SD}=0.5$} \\
\hline & & $\mathrm{E}[d]$ & SDEE & $\mathrm{E}[d]$ & SDEE & $\mathrm{E}[d]$ & SDEE \\
\hline 1 & P-128-5-1 & 0.1599 & 0.0023 & 0.1497 & 0.0069 & 0.1506 & 0.0118 \\
\hline 2 & P-128-10-1 & 0.1545 & 0.0025 & 0.1545 & 0.0074 & 0.1539 & 0.0143 \\
\hline 3 & P-128-20-1 & 0.1533 & 0.0026 & 0.1550 & 0.0103 & 0.1578 & 0.0226 \\
\hline 4 & P-128-10-1-1 & 0.1511 & 0.0031 & 0.1521 & 0.0089 & 0.1513 & 0.0146 \\
\hline 5 & P-128-10-5-1 & 0.1530 & 0.0020 & 0.1529 & 0.0065 & 0.1526 & 0.0115 \\
\hline 6 & P-128-10-10-1 & 0.1524 & 0.0028 & 0.1527 & 0.0082 & 0.1524 & 0.0205 \\
\hline 7 & 128-Cross-1 & 0.1525 & 0.0020 & 0.1524 & 0.0063 & 0.1525 & 0.0097 \\
\hline 8 & 128-Cross-2 & 0.1562 & 0.0023 & 0.1564 & 0.0071 & 0.1558 & 0.0114 \\
\hline 9 & P-256-5-1 & 0.1482 & 0.0022 & 0.1482 & 0.0069 & 0.1501 & 0.0114 \\
\hline 10 & P-256-10-1 & 0.1513 & 0.0030 & 0.1534 & 0.0091 & 0.1555 & 0.0134 \\
\hline 11 & P-256-20-1 & 0.1535 & 0.0031 & 0.1529 & 0.0097 & 0.1549 & 0.0168 \\
\hline 12 & P-256-10-1-1 & 0.1564 & 0.0020 & 0.1573 & 0.0059 & 0.1576 & 0.0099 \\
\hline 13 & P-256-10-5-1 & 0.1555 & 0.0018 & 0.1553 & 0.0056 & 0.1537 & 0.0222 \\
\hline 14 & P-256-10-10-1 & 0.1503 & 0.0009 & 0.1506 & 0.0025 & 0.1501 & 0.0118 \\
\hline 15 & 256-Cross-1 & 0.1528 & 0.0015 & 0.1527 & 0.0044 & 0.1532 & 0.0073 \\
\hline 16 & 256-Cross-2 & 0.1535 & 0.0017 & 0.1534 & 0.0051 & 0.1534 & 0.0085 \\
\hline
\end{tabular}

where only the first $N$ samples of both of the filtered signals are obtained. The filtered signals are then normalized with respect to the highest values they achieve. The two filtered signals carry a transient effect due to the filtering step by the narrow bandpass filter. The transient effect present on the two filtered signals introduces no problem to the $\mathrm{NN}$ since the constant time delay is still present between the two filtered signals and is not changed through the filtering step.

The two filtered and normalized signals are applied in three different ways to the NN. In the first method, called the parallel input form, the two signals are concatenated together to form the input vector. The resulting input vector is twice as large as either of the filtered signals. In the second method, called the difference input form, the difference between the two signals is applied to another NN system. The third method, called the single input form, uses only the filtered and normalized received signal as the NN input. The reference signal is not used as a part of the $\mathrm{NN}$ input. The motivation behind this method stems from the fact that the time delay is imbedded into the received signal. The difference and the single input forms use input vectors with lengths equal to half of the input vector length for the parallel input form, hence resulting in a large reduction in the NN size.

In the training phase, about one thousand data set with the corresponding time-delay values were introduced as training examples to the NN. For each training example, uniform random generator generated the time-delay value randomly. The time delay assume real values ranging uniformly from 0.0 to 0.5 seconds. The sampling interval was assumed to have a value of $T=0.05$ seconds. This results in time- delay values in the range of 0 to 10 sampling intervals. The damping factor $\alpha$ was generated by a uniform random generator to have any value in the range of 0.25 to 1 . The distorting noise added to the delayed signal is zero-mean Gaussian with standard deviation values chosen randomly by a uniform random generator to have any value between 0.0 and 0.5 . This step is performed so that the $\mathrm{NN}$ is trained with data sets that experienced different levels of noise.

The neural network systems used in this paper were feedforward networks. Two-layer and three-layer networks were used. Hyperbolic tangent nonlinearity was used for the hidden layer neurons, while linear transfer function was used for the output neuron. Improved version of the backpropagation training algorithm was used for training the network. It is called resilient backpropagation [11]. Riedmiller and Braun in [11] had noticed that the nonlinear transfer functions, the hyperbolic tangent and the log sigmoid, of the neurons have very small gradient values for large input values. The small gradient values result in slow convergence for the $\mathrm{NN}$ in the training phase because the backpropagation is gradient-based learning algorithm. In order to overcome this problem, the sign of the gradient was used instead of its small value to update the $\mathrm{NN}$ parameters. This resulted in a major improvement on the speed of convergence of the $\mathrm{NN}$.

Two-layer and three-layer NNs were used for the three input forms. Table 1 presents the NN which use the parallel input form, Table 2 presents the NN which use the difference input form, and Table 3 presents the NN which use the single input form. The three-layer NNs representation used for the parallel input form is denoted by $P-N-M-K-1$ notation, where $P$ denotes that the parallel input form is being used, 
TABle 2: Difference input mode (exact delay $d=0.153$ second, damping factor $\alpha=1$ ).

\begin{tabular}{|c|c|c|c|c|c|c|c|}
\hline & \multirow{2}{*}{ Neural Network } & \multicolumn{2}{|c|}{ Noise SD $=0.1$} & \multicolumn{2}{|c|}{ Noise $\mathrm{SD}=0.3$} & \multicolumn{2}{|c|}{ Noise $\mathrm{SD}=0.5$} \\
\hline & & $\mathrm{E}[d]$ & SDEE & $\mathrm{E}[d]$ & SDEE & $\mathrm{E}[d]$ & SDEE \\
\hline 1 & D-128-5-1 & 0.1533 & 0.0021 & 0.1528 & 0.0065 & 0.1519 & 0.0116 \\
\hline 2 & D-128-10-1 & 0.1525 & 0.0027 & 0.1523 & 0.0084 & 0.1523 & 0.0145 \\
\hline 3 & D-128-20-1 & 0.1515 & 0.0024 & 0.1514 & 0.0073 & 0.1516 & 0.0127 \\
\hline 4 & D-128-10-1 & 0.1546 & 0.0024 & 0.1531 & 0.0077 & 0.1509 & 0.0139 \\
\hline 5 & D-128-10-5-1 & 0.1534 & 0.0020 & 0.1530 & 0.0063 & 0.1531 & 0.0108 \\
\hline 6 & D-128-10-10-1 & 0.1537 & 0.0026 & 0.1535 & 0.0077 & 0.1541 & 0.0134 \\
\hline 7 & 128-Cross-1 & 0.1525 & 0.0020 & 0.1524 & 0.0063 & 0.1525 & 0.0097 \\
\hline 8 & 128-Cross-2 & 0.1562 & 0.0023 & 0.1564 & 0.0071 & 0.1558 & 0.0114 \\
\hline 9 & D-256-5-1 & 0.1543 & 0.0017 & 0.1545 & 0.0047 & 0.1548 & 0.0084 \\
\hline 10 & D-256-10-1 & 0.1534 & 0.0020 & 0.1529 & 0.0063 & 0.1524 & 0.0110 \\
\hline 11 & D-256-20-1 & 0.1509 & 0.0021 & 0.1555 & 0.0073 & 0.1616 & 0.0134 \\
\hline 12 & D-256-10-1-1 & 0.1570 & 0.0040 & 0.1569 & 0.0117 & 0.1563 & 0.0205 \\
\hline 13 & $D-256-10-5-1$ & 0.1523 & 0.0019 & 0.1525 & 0.0055 & 0.1530 & 0.0103 \\
\hline 14 & D-256-10-10-1 & 0.1533 & 0.0019 & 0.1534 & 0.0067 & 0.1541 & 0.0109 \\
\hline 15 & 256-Cross-1 & 0.1528 & 0.0015 & 0.1527 & 0.0044 & 0.1532 & 0.0073 \\
\hline 16 & 256-Cross-2 & 0.1535 & 0.0017 & 0.1534 & 0.0051 & 0.1534 & 0.0085 \\
\hline
\end{tabular}

TABLE 3: Single input mode (exact delay $d=0.153$ second, damping factor $\alpha=1$ ).

\begin{tabular}{|c|c|c|c|c|c|c|c|}
\hline & \multirow{2}{*}{ Neural Network } & \multicolumn{2}{|c|}{ Noise SD $=0.1$} & \multicolumn{2}{|c|}{ Noise $S D=0.3$} & \multicolumn{2}{|c|}{ Noise $S D=0.5$} \\
\hline & & $\mathrm{E}[d]$ & SDEE & $\mathrm{E}[d]$ & SDEE & $\mathrm{E}[d]$ & SDEE \\
\hline 1 & S-128-5-1 & 0.1576 & 0.0024 & 0.1582 & 0.0069 & 0.1578 & 0.0117 \\
\hline 2 & S-128-10-1 & 0.1524 & 0.0029 & 0.1528 & 0.0080 & 0.1539 & 0.0131 \\
\hline 3 & S-128-20-1 & 0.1529 & 0.0024 & 0.1523 & 0.0074 & 0.1514 & 0.0125 \\
\hline 4 & S-128-10-1-1 & 0.1542 & 0.0023 & 0.1536 & 0.0072 & 0.1521 & 0.0120 \\
\hline 5 & S-128-10-5-1 & 0.1506 & 0.0027 & 0.1520 & 0.0079 & 0.1524 & 0.0130 \\
\hline 6 & S-128-10-10-1 & 0.1503 & 0.0023 & 0.1508 & 0.0069 & 0.1511 & 0.0130 \\
\hline 7 & 128-Cross-1 & 0.1525 & 0.0020 & 0.1524 & 0.0063 & 0.1525 & 0.0097 \\
\hline 8 & 128-Cross-2 & 0.1562 & 0.0023 & 0.1564 & 0.0071 & 0.1558 & 0.0114 \\
\hline 9 & S-256-5-1 & 0.1498 & 0.0042 & 0.1503 & 0.0126 & 0.1529 & 0.0198 \\
\hline 10 & S-256-10-1 & 0.1519 & 0.0032 & 0.1543 & 0.0093 & 0.1576 & 0.0144 \\
\hline 11 & S-256-20-1 & 0.1535 & 0.0018 & 0.1537 & 0.0057 & 0.1531 & 0.0098 \\
\hline 12 & S-256-10-1-1 & 0.1527 & 0.0018 & 0.1526 & 0.0058 & 0.1519 & 0.0090 \\
\hline 13 & S-256-10-5-1 & 0.1525 & 0.0020 & 0.1521 & 0.0056 & 0.1521 & 0.0094 \\
\hline 14 & S-256-10-10-1 & 0.1528 & 0.0020 & 0.1524 & 0.0057 & 0.1523 & 0.0105 \\
\hline 15 & 256-Cross- 1 & 0.1528 & 0.0015 & 0.1527 & 0.0044 & 0.1532 & 0.0073 \\
\hline 16 & 256-Cross-2 & 0.1535 & 0.0017 & 0.1534 & 0.0051 & 0.1538 & 0.0085 \\
\hline
\end{tabular}

$N$ represents the length of each of the two input vectors, $M$ represents the number of neurons in the first hidden layer, $K$ represents the number of neurons in the second hidden layer, and the last character is the numeral 1 which is equal to the length of the output layer. The output layer is a single output neuron which outputs the estimated time delay. Similarly, the two-layer network $P-N-M-1$ represents a parallel input form structure with each of the two input vectors having a length equal to $N$, the single hidden layer consists of $M$ neurons, and the output consists of a single neuron. Similar notation 
is used for the difference input form where $P$ is replaced by $D$. Also, the single input form networks start with the letter $S$. Tables 2 and 3 show different representations of a two and a three-layer networks using difference and single input forms, respectively.

\subsection{Parallel input form}

The two filtered and normalized signals $\mathbf{s}_{f}=\left[s_{f}(0), s_{f}(1), \ldots\right.$, $\left.s_{f}(N-1)\right]$ and $\mathbf{r}_{f}=\left[r_{f}(0), r_{f}(1), \ldots, r_{f}(N-1)\right]$ are concatenated into one vector $\mathbf{x}=\left[\mathbf{s}_{f}, \mathbf{r}_{f}\right]$. The resulting vector $\mathbf{x}$ is twice the length of any of the two filtered signals. Vector $\mathbf{x}$ is applied to the NN as the training input, and the corresponding time delay $d$ as the desired delay output in seconds. Since the length of each of the filtered signals is $N$, the NN for the parallel input form has $2 \mathrm{~N}$ input nodes for its input layer.

The network represented by the P-256-10-5-1 notation found in Table 1 represents a three-layer parallel input form structure where the length of each of the two inputs is 256 . The total number of inputs for this NN is 512 . The first hidden layer consists of 10 neurons and the second hidden layer consists of 5 neurons. The output layer is a single output neuron. Also, the two-layer network P-128-20-1 in Table 1 represents a parallel input form structure with a total number of 256 inputs, 20 neurons for the single hidden layer, and one output neuron.

\subsection{Difference input form}

The difference between the two filtered signals, $\mathbf{y}=\mathbf{s}_{f}-\mathbf{r}_{f}$, is obtained and then applied to the input of the NN. The length of this vector is $N$. By using the difference input form structure, the input layer of the $\mathrm{NN}$ is reduced by a factor of two when compared to the parallel input form structure. This results in an appreciable reduction in the NN size. The three-layer difference input form with 128 input nodes, 10 neurons for the first hidden layer, 5 neurons for the second hidden layer, and one output neuron, is denoted by D-12810-5-1 as seen in Table 2 .

\subsection{Single input form}

In this method, only the filtered and normalized signal $\mathbf{r}_{f}$ is applied to the NN. The reason behind using $\mathbf{r}_{f}$ is that the time delay is embedded into the signal $\mathbf{r}_{f}$. The three-layer single input form with 128 input nodes, 10 neurons for the first hidden layer, 5 neurons for the second hidden layer, and one output neuron, is denoted by S-128-10-5-1 as seen in Table 3. All of the three input form structures are presented in Figure 1.

\section{SIMULATION RESULTS}

The simulation section consists of four sections. Sections 3.1, 3.2 , and 3.3 test the three different input forms under different input lengths and different noise levels. In those sections, the damping ratio is set equal to one. Section 3.4 tests the best performing structures obtained in the first three sections under different noise levels and different damping ratios.

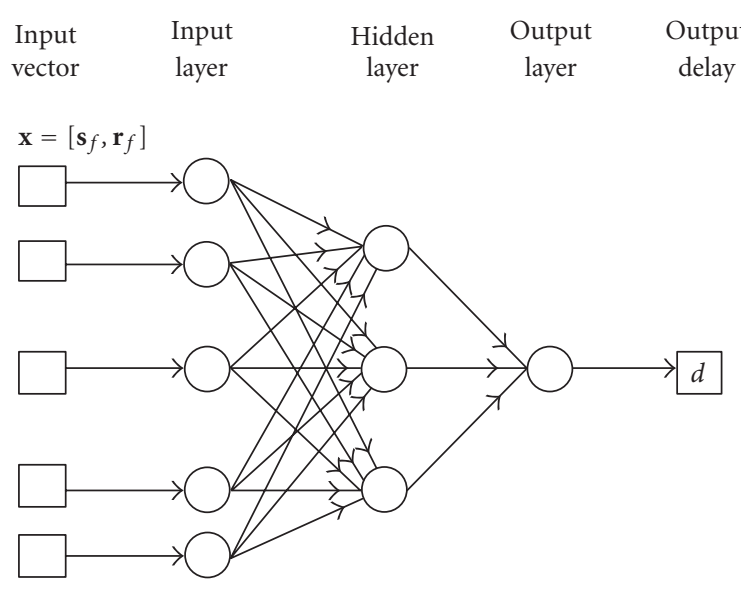

(a)

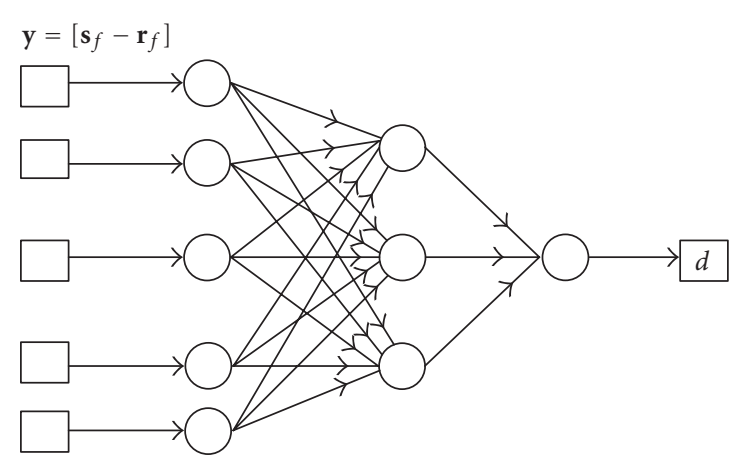

(b)

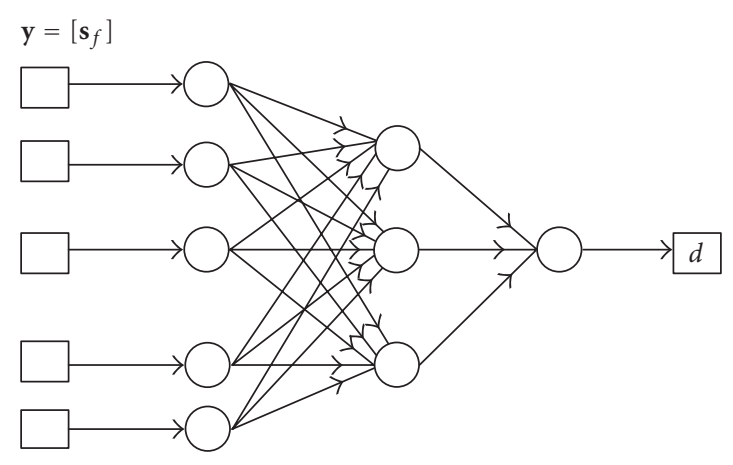

(c)

Figure 1: NNs with three input mode schemes: (a) parallel input mode with $2 N$ inputs, (b) difference input mode with $N$ inputs, and (c) single input mode with $N$ inputs.

The NN were trained with the filtered and normalized signals $\mathbf{r}_{f}$ and $\mathbf{s}_{f}$. The delayed signal was additively distorted with a zero-mean white Gaussian noise. The standard deviation of the Gaussian noise was randomly generated in the range of 0 to 0.5 , which corresponds to noise variance of 0 to 0.25 . The damping factor was randomly generated to have any value in the range of 0.25 to 1 . About 1000 data sets were used in the training phase. The signals were delayed 
by randomly selected time-delay values. A uniform random number generator generated the time-delay values that were used in the training phase. The time-delay values were chosen to have any value between 0 and 0.5 second, which corresponds to 0 to 10 sampling intervals. The frequency of the reference sinusoidal signal was chosen equal to $6 \mathrm{rad} / \mathrm{sec}$ and sampled with a sampling period of $T=0.05$ second, hence resulting in discrete reference signal $s(n)=\sin (0.3 n)$.

In the testing phase, 1000 data sets were introduced to the $\mathrm{NN}$ input. These data sets were distorted with three different noise levels. These noise levels had standard deviation values $\sigma=0.1,0.3$, and 0.5 . Two different record lengths were used, $N=128$ and $N=256$, for each noise level. Also two-layer and three-layer NNs were used. The time-delay value used for all of the testing data sets was chosen to be $0.153 \mathrm{sec}-$ ond which corresponds to 3.06 sampling intervals. The NN was tested to estimate the time delay under different damping factor values. In Sections 3.1, 3.2, and 3.3, the damping factor value was set equal to one. While in Section 3.4, the damping factor was set equal to $0.25,0.5$, and 0.75 .

For comparison reasons, delay estimates were obtained by the classical cross-correlation technique. The crosscorrelation was applied to the noise-free reference signal $s(n)$ and the delayed, damped, and noisy signal $r(n)$. This estimation process is denoted by $N$-Cross- 1 , where $N$ equals 128 or 256 samples. Also, the estimation of time delay was obtained through the cross correlation between the filtered noise-free signal $\mathbf{s}_{f}$ and the filtered delayed noisy signal $\mathbf{r}_{f}$. This estimation process is denoted by $\mathrm{N}$-Cross- 2 . The $\mathrm{N}$-Cross- 1 estimation process produced more accurate results than $\mathrm{N}$-Cross-2 when large damping factors were used. The delay estimates obtained by the NN were accurate but the cross- correlation estimates performed slightly better than the NN systems. In one case, the NN standard deviation estimation error (SDEE) was less than that obtained by the cross-correlation. It is observed that when the signal is highly attenuated, it results in time-delay estimate with large SDEE. This is explained by the statistical estimation principle which states that estimation error gets larger when signals with lower signal-to-noise ratios are used [12].

\subsection{Parallel input form}

The reference signal $\boldsymbol{s}_{f}$, the filtered and delayed noisy signal $\mathbf{r}_{f}$, and time delay $d$ were used in the training phase of the $\mathrm{NN}$. The input vector for the NN was $\mathbf{x}=\left[\mathbf{s}_{f}, \mathbf{r}_{f}\right]$. It has a length that is twice the length of any of the two signal vectors. Table 1 presents the ensemble average of the estimated time-delay values and the corresponding SDEE obtained by the NN and the cross-correlation techniques. The number of the testing data sets used was equal to one thousand. Each of these data sets was corrupted with noise having standard deviation values $\sigma=0.1,0.3$, and 0.5 . The damping factor used in all of the testing data sets was set equal to one.

It is observed from the results presented in Table 1 that the SDEE is low when low noise levels are used. Rows 1 to 6 in Table 1 correspond to data lengths of 128 samples for each of the filtered signals, where rows 1,2, and 3 correspond to the two-layer $\mathrm{NN}$ and rows 4,5 , and 6 correspond to the
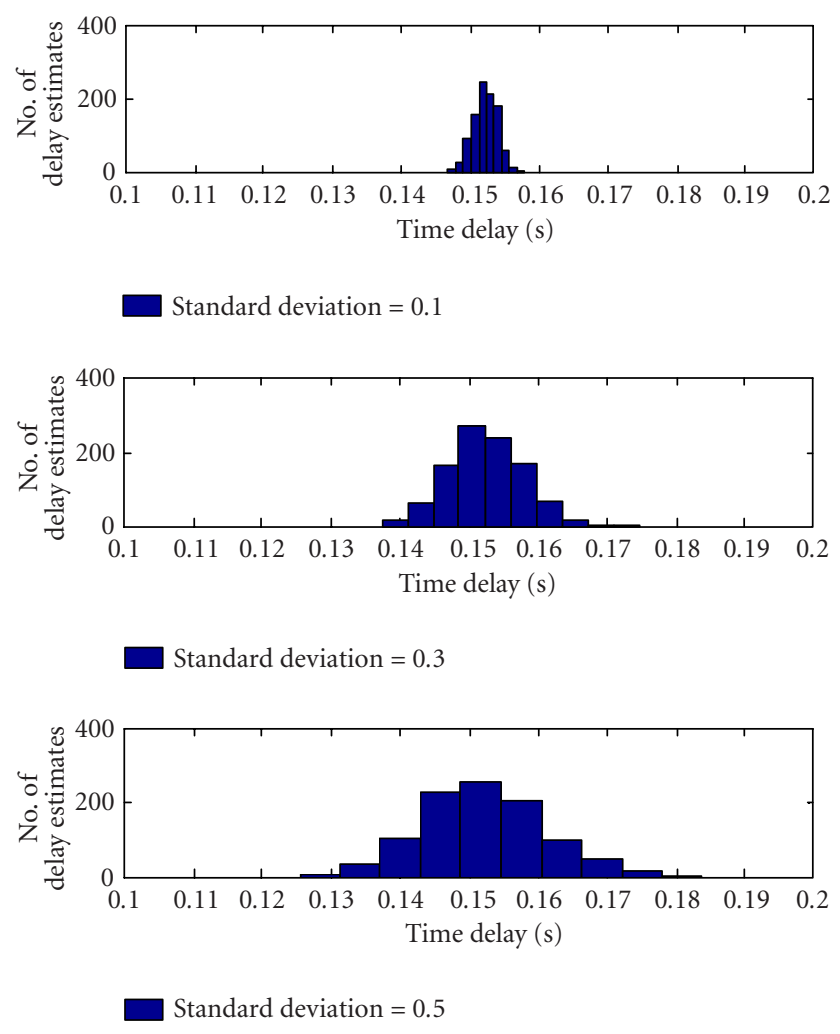

Figure 2: Histogram for the delay estimate of a delay value of 0.153 second using three different noise levels with standard deviations of $0.1,0.3$, and 0.5 . The $\mathrm{NN}$ used is the difference input mode D25610-10-1 structure.

three-layer NN. The next two rows, 7 and 8 correspond, to the 128-Cross- 1 and 128-Cross-2 estimation results, respectively. Also, rows 9 to 14 correspond to the two-layer and the three-layer NNs using 256 samples. Rows 15 and 16 correspond to the 256-Cross- 1 and the 256-Cross- 2 estimation results.

Amongst the networks using 128 samples for its inputs, the three-layer network P-128-10-5-1 produced the most accurate results in terms of the SDEE. The SDEE values are very close to those values obtained by the 128-Cross- 1 and better than those obtained by the 128-Cross-2. The two-layer network P-128-5-1 resulted in time-delay estimates with the SDEE values almost equal to the 128 -Cross- 2 technique. Amongst the networks using 256 samples, the P-256-10-101 network produced time-delay estimates with SDDE less than those obtained by the cross-correlation techniques for noise levels of 0.1 and 0.3 . For higher noise levels, the crosscorrelation techniques performed better.

\subsection{Difference input form}

The difference signal, $\mathbf{y}=\mathbf{s}_{f}-\mathbf{r}_{f}$, was applied to the NN. The damping factor used in all of the testing data was set equal to one. The estimation results are presented in Table 2. The best performing network amongst those with input record length of 128 samples is the three-layer network D-128-10-5-1. It 
TABLE 4: Best performing networks (exact delay $d=0.153$ second, damping factor $\alpha=0.25,0.5$, and 0.75 ).

\begin{tabular}{|c|c|c|c|c|c|c|}
\hline \multirow{2}{*}{ Neural Network } & \multicolumn{2}{|c|}{ Noise $\mathrm{SD}=0.1$} & \multicolumn{2}{|c|}{ Noise SD $=0.3$} & \multicolumn{2}{|c|}{ Noise $\mathrm{SD}=0.5$} \\
\hline & $\mathrm{E}[\mathrm{d}]$ & SDEE & $E[d]$ & SDEE & $\mathrm{E}[\mathrm{d}]$ & SDEE \\
\hline P-128-10-5-1 & 0.1526 & 0.0116 & 0.1571 & 0.0283 & 0.1598 & 0.0527 \\
\hline D-128-10-5-1 & 0.1522 & 0.0100 & 0.1477 & 0.0335 & 0.1339 & 0.0770 \\
\hline S-128-5-1 & 0.1532 & 0.0093 & 0.1561 & 0.0329 & 0.1597 & 0.0537 \\
\hline 128-Cross- 1 & 0.1532 & 0.0084 & 0.1416 & 0.1095 & 0.1034 & 0.5506 \\
\hline 128-Cross-2 & 0.1562 & 0.0092 & 0.1560 & 0.0295 & 0.1527 & 0.0843 \\
\hline
\end{tabular}

\begin{tabular}{l|cc|cc|rr}
\hline \multirow{2}{*}{ Neural Network } & \multicolumn{2}{|c|}{ Noise SD $=0.1$} & \multicolumn{2}{c|}{ Noise SD = 0.3 } & \multicolumn{2}{c}{ Noise SD $=0.5$} \\
\cline { 2 - 6 } & E[d] & SDEE & E[d] & SDEE & E[d] & 0.1549 \\
\hline P-128-10-5-1 & 0.1524 & 0.0060 & 0.1543 & 0.0166 & 0.0253 \\
D-128-10-5-1 & 0.1527 & 0.0051 & 0.1519 & 0.0149 & 0.1500 & 0.0267 \\
S-128-5-1 & 0.1531 & 0.0043 & 0.1532 & 0.0148 & 0.1542 & 0.1484 \\
128-Cross-1 & 0.1523 & 0.0041 & 0.1525 & 0.0122 & 0.0702 \\
128-Cross-2 & 0.1562 & 0.0045 & 0.1563 & 0.0136 & 0.1558 & 0.0233 \\
\hline
\end{tabular}

\begin{tabular}{l|cc|cc|cr}
\hline \multirow{2}{*}{ Neural Network } & \multicolumn{2}{|c|}{ Noise SD $=0.1$} & \multicolumn{2}{c|}{ Noise SD $=0.3$} & \multicolumn{2}{c}{ Noise SD $=0.5$} \\
\cline { 2 - 6 } & E[d] & SDEE & E[d] & SDEE & E[d] & 0.1546 \\
P-128-10-5-1 & 0.1522 & 0.0039 & 0.1521 & 0.0113 & 0.0181 \\
D-128-10-5-1 & 0.1529 & 0.0031 & 0.1529 & 0.0103 & 0.1511 & 0.0169 \\
S-128-5-1 & 0.1532 & 0.0028 & 0.1532 & 0.0091 & 0.1539 & 0.0163 \\
128-Cross-1 & 0.1523 & 0.0028 & 0.1526 & 0.0081 & 0.1522 & 0.1459 \\
128-Cross-2 & 0.1562 & 0.0032 & 0.1561 & 0.0092 & 0153 \\
\hline
\end{tabular}

produced the least SDEE. Also, the two-layer network D-1285-1 produced delay estimate with SDEE which are very close to that obtained by the D-128-10-5-1 network. Amongst the networks with inputs of 256 samples, the D-256-5-1 network performed the best amongst its class.

\subsection{Single input form}

For this input form, only the filtered, noisy, and delayed signal $\mathbf{r}_{f}$ was applied to the NN. The damping factor used was set equal to one. Among the first six NN structures with input length of 128, the S-128-5-1 resulted in the least SDEE. The best performing NN structures amongst those with inputs of 256 samples are the S-256-20-1 and the S-256-10-1-1 networks.

Comparing all NN results against each other, it is observed that the parallel input network performs better than the best performing networks of the difference input mode and the single input mode structures for record lengths of 256 samples. However, It must be noted that the parallel input form network has a total number of inputs equal to twice that of the other input forms. Therefore, a fair comparison should actually compare, for example, the P-128-10-1 network against the D-256-10-1 and the S-256-10-1 networks.
In such a case, it will be observed that the best performing NN structure is the difference input form.

In Figure 2, histogram plots are shown for 1000 testing data sets that were delayed by 0.153 second with noise levels $\sigma=0.1,0.3$, and 0.5 having damping factor equal to one. The histograms are produced for the network structure D-256-51. The histograms are observed to have Gaussian distribution centered at the time-delay estimate, which is near $0.153 \mathrm{sec}$ ond. It is also observed that as the noise level increases, the distribution widens which means that estimation error becomes larger.

\subsection{Best performing networks}

In this section the best performing networks in Sections 3.1, $3.2,3.3$, and 3.4 are tested under noise levels of $0.1,0.3$, and 0.5 , with record length of 128 samples, and damping factors of $0.25,0.5$, and 0.75 . Table 4 presents the simulation results for the damping factors $\alpha=0.25,0.5$, and 0.75 , respectively. The ensemble averages of the time delay and the SDEE present in Table 4 correspond to 1000 data set for each damping factor and noise level.

Comparing the resulting SDEE values for the three different input networks, it is observed that the single input 
network wins over the parallel and the difference input networks. It must be remembered that the results obtained in Table 4 correspond to damping factors $0.25,0.5$, and 0.75 . It is also observed that when low damping values are used, the SDEE estimation error gets higher. Simulation results present in Table 4 for $\alpha=0.25$ show that the NN delay estimates are more accurate than that of the 128-Cross- 1 technique for large noise levels of 0.3 and 0.5 .

\section{CONCLUSION}

NNs were utilized, for the first time, in estimating constant time delay. The NNs were trained by the filtered noisy and delayed signal and the noise-free signal. Accurate time-delay estimates were obtained by the $\mathrm{NN}$ through the testing phase. The fast processing speed of the $\mathrm{NN}$ is the main advantage of using NN for estimation of time delay. Time-delay estimates obtained through classical techniques rely on obtaining the cross correlation between the two signals and on performing interpolation to obtain the time-delay at which the peak of the cross correlation exists. These two steps are computationally demanding. The NN performs those two steps in one single pass of the data through its feedforward structure. The NN obtained accurate results and in one case produced delay estimates with smaller SDEE than that obtained by the cross-correlation techniques. Although the results obtained by the $\mathrm{NN}$ are very much encouraging, further research is still needed on the reduction of the NN size without reducing the data size. Also, it will be a major advancement if NN can be made to deal with the case in which both of the two signals are unknown. Estimating time-varying delay with $\mathrm{NN}$ is another promising subject. Estimation of time delay by $\mathrm{NN}$ has the potential of online implementation bases through the use VLSI. This will result in very fast and accurate time-delay estimates that cannot be obtained through classical techniques that demand heavy computational power.

\section{REFERENCES}

[1] G. C. Carter, Ed., "Special issue on time-delay estimation," IEEE Trans. Acoustics, Speech, and Signal Processing, vol. ASSP29, no. 3, 1981.

[2] C. H. Knapp and G. C. Carter, "The generalized correlation method for estimation of time delay," IEEE Trans. Acoustics, Speech, and Signal Processing, vol. ASSP-24, no. 4, pp. 320 327, 1976.

[3] G. C. Carter, "Coherence and time-delay estimation," Proceedings of the IEEE, vol. 75, no. 2, pp. 236-255, 1987.

[4] P. L. Feintuch, N. J. Bershad, and F. A. Reed, "Time-delay estimation using the LMS adaptive filter-dynamic behavior," IEEE Trans. Acoustics, Speech, and Signal Processing, vol. ASSP29, no. 3, pp. 571-576, 1981.

[5] Y. T. Chan, J. M. F. Riley, and J. B. Plant, "Modeling of timedelay and its application to estimation of nonstationary delays," IEEE Trans. Acoustics, Speech, and Signal Processing, vol. ASSP-29, no. 3, pp. 577-581, 1981.

[6] D. M. Etter and S. D. Stearns, "Adaptive estimation of time delays in sampled data systems," IEEE Trans. Acoustics, Speech, and Signal Processing, vol. ASSP-29, no. 3, pp. 582-587, 1981.
[7] H. C. So, P. C. Ching, and Y. T. Chan, "A new algorithm for explicit adaptation of time delay," IEEE Trans. Signal Processing, vol. 42, no. 7, pp. 1816-1820, 1994.

[8] C. During, "Recursive versus nonrecursive correlation for real-time peak detection and tracking," IEEE Trans. Signal Processing, vol. 45, no. 3, pp. 781-785, 1997.

[9] Y. T. Chan, H. C. So, and P. C. Ching, "Approximate maximum likelihood delay estimation via orthogonal wavelet transform," IEEE Trans. Signal Processing, vol. 47, no. 4, pp. 1193-1198, 1999.

[10] Z. Wang, J. Y. Cheung, Y. S. Xia, and J. D. Z. Chen, "Neural implementation of unconstrained minimum $L_{1}$-norm optimization-least absolute deviation model and its application to time delay estimation," IEEE Trans. on Circuits and Systems II: Analog and Digital Signal Processing, vol. 47, no. 11, 2000.

[11] M. Riedmiller and H. Braun, "A direct adaptive method for faster backpropagation learning: the RPROP algorithm," in Proc. IEEE Int. Conf. on Neural Networks, pp. 586-591, San Francisco, Calif, USA, 1993.

[12] H. L. V. Trees, Detection, Estimation and Modulation Theory, Part I, John Wiley \& Sons, NY, USA, 1968.

Samir Shaltaf was born in Amman, Jordan, in 1962. He received his Ph.D. degree in engineering, sensing, and signal processing, in 1992 from Michigan Technological University, Michigan, USA. He joined Amman University from 1992 to 1997. Since 1997, he has been a faculty member of the Electronic Engineering Department at Princess Sumaya University College for Technology. His research interests are in the areas of

time-delay estimation, adaptive signal processing, genetic algorithms, and control systems.

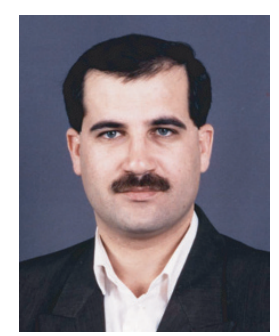

